\title{
Fingerprinting the Fractional Order Phase Transitions in AdS Black Holes
}

\author{
Mohamed Chabab* and Samir Iraoui * \\ High Energy and Astrophysics Laboratory, Physics Department, FSSM, Cadi Ayyad University, Marrakech, Morocco
}

In this paper, we have extended and deepened the study on fractional order phase transition (FPT) of a charged AdS black hole. We have carried out a detailed analysis of FPT for several AdS black hole prototypes: black hole surrounded by quintessence background, 5D Gauss-Bonnet, $D$ dimensional RN-AdS BH, and lastly Kerr black holes. We have shown that the $4 / 3$ order FPT at critical points holds for the first three black holes systems, while for Kerr black holes, the fractional order is rather $1 / 3$. These results suggest two remarkable features: Firstly $4 / 3$ order phase transition can be assumed for asymptotically AdS black holes spherical solutions; secondly the fractional order is not

OPEN ACCESS

Edited by:

Frank Franz Deppisch, University College London, United Kingdom

Reviewed by: Meng-Sen Ma,

Shanxi Datong University, China De-Cheng Zou,

Yangzhou University, China Joel Saavedra,

Pontificia Universidad Católica de valparaíso, Chile

*Correspondence:

Mohamed Chabab

mchabab@uca.ac.ma

Samir Iraoui

samir.iraoui@ced.uca.ma

Specialty section: This article was submitted to High-Energy and Astroparticle

Physics,

a section of the journal

Frontiers in Physics

Received: 22 October 2020

Accepted: 23 December 2020

Published: 14 April 2021

Citation:

Chabab M and Iraoui S (2021)

Fingerprinting the Fractional Order

Phase Transitions in AdS Black Holes.

Front. Phys. 8:620430.

doi: 10.3389/fphy.2020.620430 universal and can be affected by the geometric symmetry.

Keywords: AdS black holes, Gauss-Bonnet, Kerr black holes, phase transitions, fractional order

\section{INTRODUCTION}

Thermodynamic properties and particularly phase transitions of black holes in asymptotically Anti de-Sitter spacetime have been investigated extensively. This growing interest in phase transitions originates from their relation to holographic superconductivity in the context of the AdS/CFT correspondence [2]. Recently, many studies of black holes criticality have identified the cosmological constant with thermodynamic pressure and its conjugate quantity with thermodynamic volume [3-6]. Furthermore the inclusion of the $P-V$ term in the first law of thermodynamics has led to identification of the black hole mass with the enthalpy of the event horizon $(H \equiv M)$ [7]. Then, the analogy with the Van de Waals $P-V$ criticality has been established [8] and the first- and secondorder phase transitions have been readily found [6]. Generalization to thermodynamics structure of Reissner-Nordström black holes surrounded by quintessence has also been carried out [9-13].

Originally outlined by Ehrenfest who used the discontinuities in derivatives of the free energy to probe and classify thermodynamic phase transitions, Ehrenfest's classification scheme is based on the experimental observation of the density contrast between coexisting phases and latent heat during a phase transition. When a system undergoes a phase transition at a critical temperature, Ehrenfest defines its order of phase transition as the smallest integer $n \geq 1$ such that the $n$ - th derivative of $g(T)$ has a discontinuity at the critical point. More formally this criterion generally reads as

$$
\lim _{T \rightarrow T_{c}^{+}} \frac{\mathrm{d}^{n} g(T)}{\mathrm{d} T^{n}}=A^{+} \neq A^{-}=\lim _{T \rightarrow T_{c}^{-}} \frac{\mathrm{d}^{n} g(T)}{\mathrm{d} T^{n}},
$$

where $g(T)$ denotes the free energy as a function of temperature $T$.

Hawking-Page phase transition is an example of first-order transition. The critical point is commonly presented as a witness of second-order phase transition in the sense of Ehrenfest. A much more general classification scheme based on fractional derivatives, viz. a derivative with noninteger order, along arbitrary curves in the thermodynamic state space, has been revealed [15-17]. Fractional phase transitions (FPT) go back to the work of Nagle on dipalmitoyl lecithin (DPL) system [18], 
where it has been observed that the order $\leftrightarrow$ disorder phase transition is neither of first nor of second type but is rather of $3 / 2$ order (see also Ref. 19). Besides, 4/3 order FPTs have also been found in Van der Waals fluids [14].

The generalized Ehrenfest classification holds for all thermodynamic systems having phase structures, including AdS black holes with analogous behavior to Van der Waals gases. Indeed, a first study recently performed by $\mathrm{Ma}$ [1] showed that RN-AdS black holes can undergo 4/3 order FPT. In this paper, we aim to explore further this issue and see whether this fractional order holds for any AdS black hole solution, as in VdW systems. To this end, we consider four prototypes: First the charged AdS black hole surrounded by quintessence field to show the effect of an external source. Second, we use a $D$ dimensional RN-AdS and fivedimensional Gauss-Bonnet-AdS black hole to probe whether higher spacetime dimensions, $d>4$, and higher derivative corrections can affect and modify the FPT order near the critical point. At last, we also study FPT beyond spherical symmetric static black holes through Kerr black holes and verify the role played by the geometric symmetry when dealing with FPT.

This paper is organized as follows. In the next section, first we present the following AdS black hole solutions: Kiselev, $D$ dimensional RN-AdS, and 5D Gauss-Bonnet solution, and their corresponding thermodynamic quantities. Then, we study their fractional phase transitions at critical points via the Caputo derivative of Gibbs free energy. Section 3 is devoted to the analysis of Kerr-AdS black hole and derivation of its FPT order. Our conclusion is drawn in the last section.

\section{THERMODYNAMIC CRITICALITY AND FRACTIONAL ORDER PHASE TRANSITIONS OF ADS BLACK HOLES WITH SPHERICAL SYMMETRY}

In this section, we consider three spherical symmetric solutions of static asymptotically AdS black holes, namely: a charged AdS $\mathrm{BH}$ surrounded by quintessence, $D$ dimensional RN-AdS BH, and a 5D Gauss-Bonnet AdS black holes. We first calculate their thermodynamic quantities and then describe the behaviors of corresponding Gibbs free energy and their fractional derivatives nearby the critical points. Our objective is to probe whether the extra factors, as quintessence parameter, higher dimensions, and/ or higher derivatives in Gauss-Bonnet term parameters, could affect the FPT order.

\subsection{Charged AdS Black Hole Surrounded by Quintessence}

From high precision astronomical observations, it has been shown that the Universe is currently undergoing a phase of accelerated expansion [20,21], which might be due to dark energy acting as a repulsive gravity. A possible origin of this phenomenon could come from the so-called quintessence field, which obeys an equation of state formulated through the relation between negative pressure and energy density as $p=\omega_{q} \rho_{q}$, where the quintessence parameter is constrained as $-1<\omega<-1 / 3$ [22].

We consider the Kiselev solution of four-dimensional charged AdS black holes surrounded by quintessence,

$$
f(r)=1-\frac{2 M}{r}+\frac{Q^{2}}{r^{2}}-\frac{\Lambda}{3} r^{2}-\frac{\alpha}{r^{3 \omega_{q}+1}},
$$

where $a$ represents a positive normalization parameter, while $M$ and $Q$ are the mass and electric charge of the black hole, respectively. As usual, we treat the cosmological constant as a dynamical pressure of the black hole [7],

$$
P=-\frac{\Lambda}{8 \pi}
$$

The Hawking temperature related to the surface gravity via the formula $2 \pi T=\kappa$ is then expressed as [13]

$$
T=\frac{1}{4 \pi}\left[\frac{1}{r_{h}}-\frac{Q^{2}}{r_{h}^{3}}+\frac{3 \omega_{q} \alpha}{r_{h}^{3 \omega_{q}+2}}+8 \pi r_{h} P\right],
$$

where the horizon $r_{h}$ is determined from the condition $f\left(r_{h}\right)=0$. The equation of state for the charged AdS black hole surrounded by quintessence is given by

$$
P=\frac{2 \pi T}{2 \pi v}-\frac{1}{2 \pi v^{2}}+\frac{2 Q^{2}}{\pi v^{4}}-\frac{3 \times 2^{1+3 \omega_{q}} \alpha \omega_{q}}{2 \pi v^{3 \omega_{q}+3}},
$$

where the specific volume $v$ associated with the fluid volume is related to the horizon radius since $v=2 r_{h}$. The first law of black hole thermodynamics in the extended phase space can be written as [11]

$$
d M=T \mathrm{~d} S+V \mathrm{~d} P+\Phi \mathrm{d} Q+\mathcal{Q} \mathrm{d} \alpha,
$$

where the conjugate quantities of the parameters $P, Q$, and $a$ read, respectively, as

$$
V=\frac{4 \pi r_{h}^{3}}{3}, \quad \Phi=\frac{Q}{r_{h}}, \quad \mathcal{Q}=-\frac{1}{2 r_{h}^{3 \omega_{q}}},
$$

while the Smarr relation is formulated by

$$
M=2 T S-2 P V+\Phi Q+\left(3 \omega_{q}+1\right) \mathcal{Q} \alpha .
$$

Thereafter, without loss of generality, we will set $\omega_{q}$ to the value $\omega_{q}=-2 / 3$. In this case, we can determine the critical point analytically as

$$
T_{c}=\frac{\sqrt{6}-9 Q \alpha}{18 \pi Q}, \quad v_{c}=2 \sqrt{6} Q, \quad P_{c}=\frac{1}{96 \pi Q^{2}} .
$$

For subsequent analysis, it is more appropriate to introduce the following variables:

$$
p=\frac{P-P_{c}}{P_{c}}, \quad t=\frac{T-T_{c}}{T_{c}}, \quad v=\frac{v-v_{c}}{v_{c}} .
$$

Thus with the new set of variables $(t, p, v)$, the critical point lies at $(t=p=v=0)$, while the equation of state reduces to a quartic equation in $\nu$, 

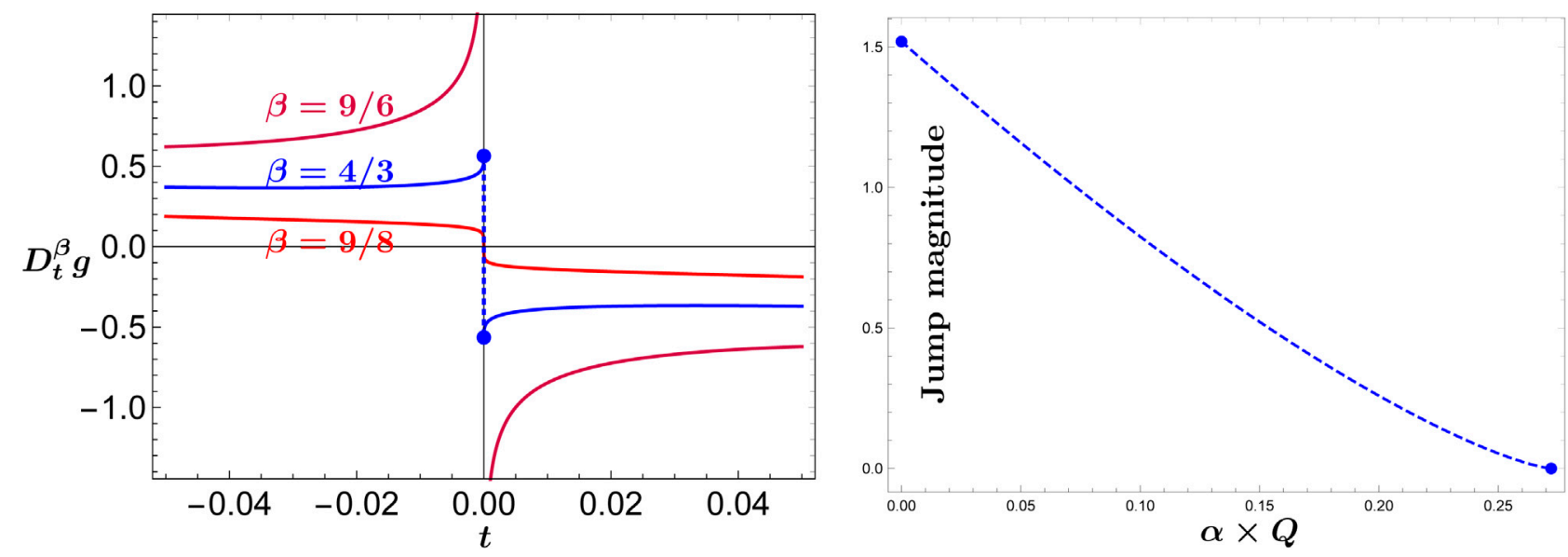

FIGURE 1 | Left panel: The behavior of fractional derivatives $D_{t}^{\beta} g$ for $\beta$ equal to 9/6 (purple line), $9 / 8$ (red line), and 4/3 (blue line) near the critical point of charged AdS black hole surrounded by quintessence. Input: $Q \alpha=0.55$. Right panel: Jump magnitude near the critical point as a function of a for $\beta=4 / 3$.

$$
3 p(\nu+1)^{4}+4 t(\nu+1)^{3}(3 \sqrt{6} Q \alpha-2)+v^{3}(3 v+4)=0 .
$$

Besides, the rescaled Gibbs free energy now takes the form

$$
g(t, p)=\frac{8-v^{4}-4 v^{3}+8 v-(v+1)^{4} p}{4 \sqrt{6}(v+1)}
$$

Next we probe the behavior of Gibbs free energy $g(t, p)$ and its fractional derivatives near critical point. First we use the expansion series of $v(t, p)$ derived from Eq. 11 and substitute it into the $g(t, p)$. The resulting expression reads as

$$
\begin{aligned}
& g(t, p) \approx\left[\sqrt{\frac{2}{3}}+\frac{p}{2 \sqrt{6}}+\ldots\right] \\
& -\left[\sqrt{\frac{2}{3}}-3 Q \alpha-\frac{(2-3 \sqrt{6} Q \alpha)}{6^{1 / 6}}\left(p^{1 / 3}-\frac{19}{4 \times 6^{2 / 3}} p^{2 / 3}\right)+\ldots\right] t \\
& -\left[\frac{2^{1 / 6}\left(2-6 \sqrt{6} Q \alpha+27 Q^{2} \alpha^{2}\right)}{9 \times 3^{1 / 6}}\left(\frac{2^{5 / 3}}{p^{2 / 3}}-\frac{13}{9^{1 / 3} p^{1 / 3}}\right)+\ldots\right] t^{2} \\
& +\mathcal{O}\left[t^{3}\right] .
\end{aligned}
$$

The above equation can well be expressed in the Taylor series of $t$ and written in a simple form as

$$
g(t, p)=A(p)+B(p) t+D(p) t^{2}+\mathcal{O}\left[t^{3}\right] .
$$

Several definitions of fractional derivatives exist in literature [23]. Here we rely on Caputo definition which enables easy use of conventional boundaries and initial conditions [24]:

$$
D_{t}^{\beta} g(t)=\frac{1}{\Gamma(n-\beta)} \int_{0}^{t}(t-\tau)^{n-\beta-1} \frac{\partial^{n} g(\tau)}{\partial \tau^{n}} \mathrm{~d} \tau, \quad n-1<\beta<n,
$$

where $\beta$ is the order of derivative and $n$ an integer. As a result of calculation we get

$$
\left.D_{t}^{\beta} g(t, p)\right|_{p}=\left\{\begin{array}{cc}
\frac{2 D(p)}{\Gamma(3-\beta)} t^{2-\beta}+\frac{(\beta-2) B(p)}{\Gamma(3-\beta)} t^{1-\beta}, & t>0 ; 0<\beta<1, \\
-\frac{2 D(p)}{\Gamma(3-\beta)}|t|^{2-\beta}-\frac{(\beta-2) B(p)}{\Gamma(3-\beta)}|t|^{2-\beta}, & t<0 ; 0<\beta<1, \\
\frac{2 D(p)}{\Gamma(3-\beta)} t^{2-\beta}, & t>0 ; 1<\beta<2, \\
-\frac{2 D(p)}{\Gamma(3-\beta)}|t|^{2-\beta}, & t<0 ; 1<\beta<2 .
\end{array}\right.
$$

According to Refs. 11 and 13, the equation of state near the critical point $(t \ll 1, v \ll 1)$ simplifies to

$$
p \approx k t+O\left[t^{2}, t \nu\right]
$$

where the slope $k$ is given by

$$
k=\frac{T_{c}}{P_{c} v_{c}}=\frac{8}{3}-4 \sqrt{6} Q \alpha .
$$

Therefore, we can calculate the values of $D_{t}^{\beta} g(t, p)$ for $1<\beta<2$ in the limit $(t \rightarrow 0, p \rightarrow 0)$ by substituting Eq. 16 into Eq. 15. Thus, we obtain

$$
\lim _{t \rightarrow 0^{ \pm}} D_{t}^{\beta} g(t, p)=\left\{\begin{array}{cc}
0 & \text { for } \beta<4 / 3, \\
\mp \frac{(2-3 \sqrt{6} \alpha Q)^{4 / 3}}{\sqrt{6} \Gamma\left(\frac{2}{3}\right)} & \text { for } \beta=4 / 3, \\
\mp \infty & \text { for } \beta>4 / 3 .
\end{array}\right.
$$

Obviously, for $\beta=4 / 3$ case, we see a jump discontinuity,

$$
\lim _{t \rightarrow 0^{-}} D_{t}^{\beta} g \neq \lim _{t \rightarrow 0^{+}} D_{t}^{\beta} g \text {. }
$$

When $\beta>4 / 3$, the $\beta$-order fractional derivatives of the Gibbs free energy diverge. Hence, as for 4D RN-AdS black holes [1], the FPT order of the RN-AdS black hole surrounded by quintessence is 
$\beta=4 / 3$. This is clearly illustrated by the left panel of Figure 1 where the fractional derivative $D_{t}^{\beta} g$ is plotted as a function of the reduced temperature $t$ for several values of $\beta: \beta=9 / 6,9 / 8$, and $4 / 3$ near the critical point. One can see that this plot is continuous at $T_{c}$ for $\beta<4 / 3$ and becomes progressively steeper as $\beta$ approaches $4 / 3$ but behaves as discontinuous at $T_{c}$ for $\beta=4 / 3$ and fully divergent once $\beta>4 / 3$. It is also worth to notice the behavior of the magnitude $\frac{(4-6 \sqrt{6} \alpha Q)^{4 / 3}}{\sqrt{6} \Gamma\left(\frac{2}{3}\right)}$ for $\beta=4 / 3$ shown in Eq. 18. This magnitude monotonically decreases as far as $a$ increases and becomes $\frac{4^{4 / 3}}{\sqrt{6} \Gamma\left(\frac{2}{3}\right)}$ when $\alpha \rightarrow 0$, whereas it fades away in the limit $\alpha \rightarrow \sqrt{6} /(9 Q)$, that is, $T_{c} \rightarrow 0$, as shown by the right panel of Figure 1 .

This result clearly shows that, for spherical symmetric AdS black hole in quintessence background, the FPT still stands at 4/3 order. Therefore, the fractional phase transition is not affected by the external quintessence field surrounding the charged AdS black hole.

\subsection{Charged AdS Black Holes in Higher Dimensions}

Now we consider a $D$ dimensional charged AdS black hole to check whether higher dimension $D$ deflects the fractional order of the phase transition from $4 / 3$. For this case, the metric is given by

$$
\begin{gathered}
d s^{2}=-f \mathrm{~d} t^{2}+\frac{\mathrm{d} r^{2}}{f}+r^{2} \mathrm{~d} \Omega_{D-2}^{2}, \\
f(r)=1-\frac{m}{r^{D-3}}+\frac{q^{2}}{r^{2(D-3)}}-\frac{2 \Lambda r^{2}}{(D-2)(D-1)},
\end{gathered}
$$

$m$ and $q$ are related to the $\mathrm{ADM}$ mass $M$, while $Q$ stands for the black hole charge [25]:

$$
M=\frac{D-2}{16 \pi} \omega_{D-2} m, \quad Q=\frac{\sqrt{2(D-2)(D-3)}}{8 \pi} \omega_{D-2} q
$$

with $\omega_{D}$ veing the volume of the unit $D$-sphere.

Thermodynamics of higher dimension black holes has been investigated in Ref. 26 as well as their critical behaviors revealed as phase transition between small and large black holes. The critical points were derived at

$$
\begin{aligned}
v_{c} & =\frac{4}{D-2}\left[q^{2}(D-2)(2 D-5)\right]^{\frac{1}{2(D-3)}}, \\
T_{c} & =\frac{(D-2)(d-3)^{2}}{(2 D-5)} \frac{1}{4 \pi v_{c}} \\
P_{c} & =\left(\frac{D-3}{D-2}\right)^{2} \frac{1}{\pi v_{c}^{2}}
\end{aligned}
$$

where the specific volume is given, in the geometric units, by $v=\frac{4}{D-2} r_{h}$. As previously once we use the reduced variable, the equation of state transforms to the following general form:

$$
\begin{aligned}
4 t= & \frac{2 D-5}{(D-3)(v+1)}-\frac{(\nu+1)^{5-2 D}}{6+D(D-5)}+\frac{D(2 D-5)(\nu+1)(p+1)}{(D-2)^{2}} \\
& -\frac{2(2 D-5)(\nu+1)(p+1)}{(D-2)^{2}}-4 .
\end{aligned}
$$

TABLE 1 | Limits and fractional order phase transition at the critical point for $D$ dimensional RN-AdS BH.

\begin{tabular}{lcc}
\hline Dimension $\boldsymbol{d}$ & Order FPT & $\lim _{\mathbf{t} \rightarrow \mathbf{0}^{ \pm}} \mathbf{D}_{\mathbf{t}}^{\beta} \mathbf{g}(\mathbf{t}, \mathbf{p})$ \\
\hline$D=4$ & $\beta=4 / 3$ & $\mp \frac{22^{5 / 6}}{3 \sqrt{3} \Gamma\left(\frac{5}{3}\right)}$ \\
$D=5$ & $\beta=4 / 3$ & $\mp \frac{8 \sqrt{2}}{\sqrt{3} 5^{5 / 6} \Gamma\left(\frac{5}{3}\right)}$ \\
$D=6$ & $\beta=4 / 3$ & $\mp \frac{12 \sqrt{6}}{7^{5 / 6} \Gamma\left(\frac{5}{3}\right)}$ \\
$D=7$ & $\beta=4 / 3$ & $\mp \frac{32 \sqrt[3]{3} \sqrt{5}}{9 \Gamma\left(\frac{5}{3}\right)}$ \\
$D=8$ & $\beta=4 / 3$ & $\mp \frac{5\left(\frac{2}{11}\right)^{5 / 6}}{\sqrt{3} \Gamma\left(\frac{5}{3}\right)}$ \\
$D=9$ & $\beta=4 / 3$ & $\mp \frac{24 \sqrt[3]{6} \sqrt{7}}{13^{5 / 6} \Gamma\left(\frac{5}{5}\right)}$ \\
$D=10$ & $\beta=4 / 3$ & $\mp \frac{19\left(\frac{5}{5}\right)^{5 / 6}}{3 \sqrt{3} \Gamma\left(\frac{5}{3}\right)}$ \\
\hline
\end{tabular}

The rescaled Gibbs free energy is

$$
\begin{gathered}
g(t, p)=\frac{(5-2 D)^{2}(\nu+1)^{3-D}}{2[(D-2)(2 D-5)]^{3 / 2}}+\frac{1}{2} \sqrt{\frac{2 D-5}{D-2}}(\nu+1)^{D-3} \\
-\sqrt{\frac{2 D-5}{(D-2)^{3}}} \frac{(D-3)^{2}}{2(D-1)}(p+1)(\nu+1)^{D-1},
\end{gathered}
$$

Note that for $D=4$ the results obtained in Ref. 1 for $\mathrm{RN}-\mathrm{AdS}_{4} \mathrm{BH}$ are recovered.

Next step, we solve Eq. 24 and expand the Gibbs free energy for each dimension spacetime $D>5$. As illustration, consider $D=6$; then the equation for $g(t, p)$ near the critical point reduced to

$$
\begin{aligned}
& g(t, p) \approx\left(\frac{\sqrt{7}}{5}+\frac{9 \sqrt{7} p}{20}+\ldots\right)+\left(-\frac{9}{\sqrt{7}}+\frac{18 \sqrt[3]{3} p^{1 / 3}}{\sqrt{7}}-\frac{15}{4}\left(3^{2 / 3} \sqrt{7}\right) p^{2 / 3}+\frac{60677 p}{768 \sqrt{7}}+\ldots\right) t \\
& +\left(-\frac{48 \sqrt[3]{3}}{7 \sqrt{7} p^{2 / 3}}+\frac{1163^{2 / 3}}{7 \sqrt{7} p^{1 / 3}}+\ldots\right) t^{2}+\mathcal{O}\left[t^{3}\right]
\end{aligned},
$$

Since the equation of state near the critical point behaves as $p \approx \frac{4 D-8}{2 D-5} t$, and for $t$ approaching 0 , we find that the fractional derivative of $g(t, p)$ is discontinuous function,

$$
\lim _{t \rightarrow 0^{ \pm}} D_{t}^{\beta} g(t, p)=\left\{\begin{array}{cc}
0 & \text { for } \beta<4 / 3, \\
\mp \frac{12 \sqrt[3]{6}}{7^{5 / 6} \Gamma\left(\frac{5}{3}\right)} & \text { for } \beta=4 / 3, \\
\mp \infty & \text { for } \beta>4 / 3,
\end{array}\right.
$$

with a discontinuity taking place at $\beta=4 / 3$. We summarized the calculation results for all dimensions $D=4-10$ in Table 1 .

Table 1 decidedly shows that, whatever the dimension $D$ of the $\mathrm{RN}$-AdS black hole, the fractional order of phase transition near the critical point arises decidedly at $\beta=5 / 3$, with the jump magnitude increasing as $D$ dimension gets larger.

\subsection{D Gauss-Bonnet-AdS Black Hole}

First we briefly introduce the action of Gauss-Bonnet black hole and its main thermodynamic features.

The action of this theory reads as 


$$
\mathcal{S}_{L}=\int \mathrm{d}^{D} x \sqrt{-g}\left(R-2 \Lambda+\alpha^{\prime} \mathcal{L}_{2}\right)
$$

$\mathcal{L}_{2} \quad$ represents the Gauss-Bonnet term $\mathcal{L}_{2}=R_{\mu \nu \lambda \sigma} R^{\mu \nu \lambda \sigma}-4 R_{\mu \nu} R^{\mu \nu}+R^{2}$, with the Riemann curvature tensor $R_{\mu \nu \lambda \sigma}$ and Ricci tensor $R_{\mu \nu}$, while $R$ is the Ricci scalar. $\alpha^{\prime}$ is a dimensionless coupling constant.

A spherically symmetric static solution of this theory has been derived, and the metric function determined through solving the real roots of a polynomial equation [27, 28].

It is founded to seek a connection between the higher derivatives in Gauss-Bonnet term and the phase transition, checking whether it affects the FPT's order. Here, we use as prototype $D=5$ Gauss-Bonnet-AdS black holes, which exhibit a critical behavior featured via small/large phase transition, while for $D \geq 6$ no phase structure has been unveiled $[29,30]$. The exact solution describing this black hole is given by Ref. 28 .

$$
f_{G B}(r)=1+\frac{r^{2}}{2 \tilde{\alpha}}\left(1-\sqrt{1-\frac{16 \pi P \tilde{\alpha}}{3}+\frac{32 M \tilde{\alpha}}{3 \pi r^{4}}}\right),
$$

where $\tilde{\alpha}$ is a Gauss-Bonnet coupling constant. In the extended phase space, the equation of states reads as

$$
P=\frac{32 \tilde{\alpha} T}{9 v^{3}}+\frac{T}{v}-\frac{2}{3 \pi v^{2}},
$$

where the specific volume is $v=\frac{4}{3} r_{h}$. The thermodynamic critical point coordinates are

$$
P_{c}=\frac{1}{48 \pi \tilde{\alpha}}, \quad T_{c}=\frac{1}{2 \sqrt{6} \pi \sqrt{\tilde{\alpha}}}, \quad v_{c}=4 \sqrt{\frac{2 \tilde{\alpha}}{3}},
$$

while the rescaled Gibbs free energy $(G / \tilde{\alpha})$ reads as

$$
g(t, p)=\frac{v^{3}(\nu+2)^{3}+(\nu(\nu+2)+4)(\nu+1)^{4} p}{8(3 v(\nu+2)+4)} .
$$

As previous analysis, since the equation of state near the critical point [29] is reduced to $p \approx 4 t+O\left[t^{2}, t v\right]$, the fractional derivative of $g$ can be written as the following form:

$$
\lim _{t \rightarrow 0^{ \pm}} D_{t}^{\beta} g(t, p)=\left\{\begin{array}{cc}
0 & \text { for } \beta<4 / 3 \\
\mp \frac{2^{5 / 3}}{\Gamma\left(\frac{5}{3}\right)} \pi & \text { for } \beta=4 / 3 . \\
\mp \infty & \text { for } \beta>4 / 3
\end{array} .\right.
$$

Again, we find that the phase transition near the critical point is fractional and happens at order $4 / 3$, which suggest that neither the spacetime dimension nor the higher derivative corrections introduced by means of the Gauss-Bonnet term can alter the fractional order phase transition at the critical point. Hence we assume that FPT is located at 4/3 order, whatever the external factors (space dimension, surrounding background) as far as the AdS black hole solution possesses a spherical symmetry.

The next legitimate question is what about axisymmetric black hole solutions? Is the $4 / 3$ order of FPT universal? we attempt to respond to this issue in the subsequent section using Kerr-AdS black hole as prototype.

\section{KERR-ADS BLACK HOLES}

The Kerr solution is the only known family of exact solutions which could present the stationary axisymmetric field outside a rotating massive object.

By using Boyer-Lindquist coordinates, the Kerr asymptotically AdS black hole solutions can read as [31-33]

$$
\begin{aligned}
d s^{2}= & -\frac{\Delta}{\rho^{2}}\left[d t-\frac{a \sin ^{2} \theta}{\Xi} d \varphi\right]^{2}+\frac{\rho^{2}}{\Delta} d r^{2}+\frac{\rho^{2}}{S} d \theta^{2} \\
& +\frac{S \sin ^{2} \theta}{\rho^{2}}\left[a d t-\frac{r^{2}+a^{2}}{\Xi} d \varphi\right]^{2},
\end{aligned}
$$

where

$$
\begin{gathered}
\rho^{2}=r^{2}+a^{2} \cos ^{2} \theta, \Xi=1-\frac{a^{2}}{l^{2}}, S=1-\frac{a^{2}}{l^{2}} \cos ^{2} \theta, \\
\Delta=\left(r^{2}+a^{2}\right)\left(1+\frac{r^{2}}{l^{2}}\right)-2 m r .
\end{gathered}
$$

The mass $M$ and the angular momentum $J$ are related to the parameters $m$ and $a$ as follows:

$$
M=\frac{m}{\Xi^{2}}, \quad J=\frac{a m}{\Xi^{2}}
$$

Knowing that the pressure is still given by Eq. 3 and neglecting all terms of higher order in $J$, the equation of state in terms of temperature, specific volume, and the angular momentum reads as [26]

$$
P=\frac{T}{v}-\frac{1}{2 \pi v^{2}}+\frac{48}{\pi v^{6}} J^{2}+\mathcal{O}\left[J^{4}\right]
$$

where the specific volume associated with the Kerr black hole is given by

$$
v=2\left(\frac{3 V}{4 \pi}\right)^{1 / 3}=2 r_{+}+\frac{12}{r_{+}^{3}\left(3+8 \pi r_{+}^{2} P\right)} J^{2} .
$$

The critical point occurs at

$$
v_{c}=2 \times 90^{1 / 4} \sqrt{J}, \quad T_{c}=\frac{90^{3 / 4}}{225 \pi} \frac{1}{\sqrt{J}}, \quad P_{c}=\frac{1}{12 \sqrt{90} \pi} \frac{1}{J} .
$$

From the approximation used previously, we derive the expression of rescaled Gibbs free energy in terms of the dimensionless parameters given in Eq. 10,

$$
\begin{aligned}
g(t, p) & =G / \sqrt{J} \\
& =\frac{\sqrt[4]{5}\left(-12 \sqrt[4]{10} \sqrt{J}(\nu+1)^{7}(p+1)+9 \sqrt{3}(\nu+1)^{4}+\sqrt{3}\right)}{182^{3 / 4}(\nu+1)^{3}},
\end{aligned}
$$

and the reduced equation of state 


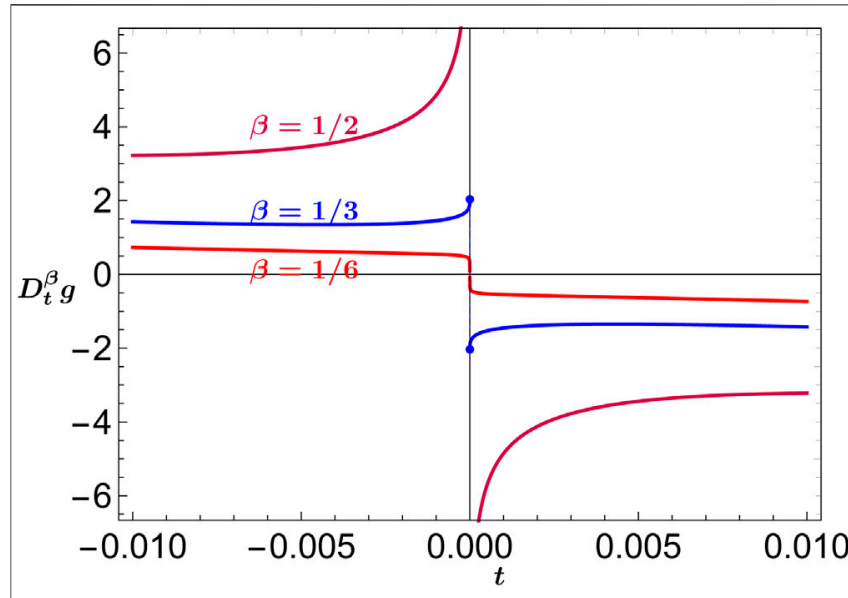

FIGURE 2 | The behaviors of fractional derivatives $D_{t}^{\beta} g$ for $\beta$ equal to $1 / 2$ (purple line), 1/6 (red line), and 1/3 (blue line) near the critical point of Kerr-AdS black hole, with $J=1$.

$$
v^{3}\left(10 v^{3}+36 v^{2}+45 v+20\right)+10(v+1)^{6} p-24(v+1)^{5} t=0 .
$$

Then, using Eqs 40 and 41, one can readily derive the phase transition order near the critical point. Indeed, $t$-expansion of the rescaled Gibbs free energy becomes

$$
\begin{aligned}
& g(t, p) \approx C_{1}+\left[C_{2}+\frac{C_{3}}{p^{2 / 3}}+\frac{C_{4}}{p^{1 / 3}}+\ldots\right] t+\left[C_{5}+\frac{C_{6}}{p^{5 / 3}}+\frac{C_{7}}{p^{4 / 3}}+\frac{C_{8}}{p^{2 / 3}}\right. \\
& \left.++\frac{C_{9}}{p^{1 / 3}}+\ldots\right] t^{2}+\mathcal{O}\left[t^{3}\right],
\end{aligned}
$$

where the coefficients $C_{i}$ are functions of $J$. Moreover, the pressure behaves as $p \approx \frac{12}{5} t$ near the critical point. Using Eq. 15, we obtain the fractional derivative of $g(t, p)$ with $0<\beta<2$ in the limits $t \rightarrow 0$ and $p \rightarrow 0$,

$$
\lim _{t \rightarrow 0^{ \pm}} D_{t}^{\beta} g(t, p)=\left\{\begin{array}{cc}
0 & \text { for } \beta<1 / 3 \\
\pm \frac{5\left(\frac{10}{3}\right)^{2 / 3}\left(2 C_{3}-C_{6}\right)}{9 \Gamma\left(\frac{11}{3}\right)} & \text { for } \beta=1 / 3 \\
\mp \infty & \text { for } \beta>1 / 3,
\end{array}\right.
$$

with

$$
\begin{aligned}
& C_{3}=\frac{\sqrt[6]{2}\left(2^{3 / 4} \sqrt{3}-16 \sqrt[4]{5} \sqrt{J}\right)}{3 \times 5^{3 / 4}} \\
& C_{6}=\frac{4 \sqrt[6]{2}\left(2^{3 / 4} \sqrt{3}-16 \sqrt[4]{5} \sqrt{J}\right)}{15 \times 5^{3 / 4}}
\end{aligned}
$$

The fractional order phase transition of Kerr-AdS black hole clearly arises at $\beta=1 / 3$. Figure 2 illustrates the fractional derivative $D_{t}^{\beta} g$ for $\beta$ equals to $1 / 2,1 / 6$, and $1 / 3$ near the critical point.

Therefore, near the critical point, we see that fractional order of the phase transition is no longer $\beta=4 / 3$. This may suggest $4 / 3$ order FPT is not universal and only holds for static black holes with spherical symmetry.

\section{CONCLUSION}

Summarizing, in this paper we have studied the continuous thermodynamic phase transitions of AdS black holes according to the generalized Ehrenfest classification. By using the Caputo fractional derivatives of thermodynamic potentials for a charged black hole surrounded by quintessence and $5 \mathrm{D}$ Gauss-Bonnet and $\mathrm{RN}-\mathrm{AdS}_{D}$ black holes, we find that the fractional derivatives of the Gibbs free energy are always discontinuous at the critical point for $\beta=4 / 3$ order and diverge when $\beta>4 / 3$. These results suggest that the $4 / 3$ order phase transition is robust and holds as far one deals with static black hole with spherical symmetry. However, this feature is not universal and fails for axisymmetric solutions, as demonstrated for Kerr black hole where the phase transition happens at $\beta=1 / 3$ order. Nevertheless, further investigations of other black holes configurations are required to consolidate these findings and establish a more involved classification of thermodynamic phase transitions.

\section{DATA AVAILABILITY STATEMENT}

The original contributions presented in the study are included in the article/Supplementary Material, further inquiries can be directed to the corresponding authors.

\section{AUTHOR CONTRIBUTIONS}

All authors listed have made a substantial, direct, and intellectual contribution to the work and approved it for publication.

\section{ACKNOWLEDGMENTS}

This manuscript has been released as a preprint at arXiv database [34]. 


\section{REFERENCES}

1. Ma MS. Fractional-order phase transition of charged AdS black holes. Phys Lett $B$ (2019) 795:490-495. doi:10.1016/j.physletb.2019.06.054

2. Aharony O, Gubser SS, Maldacena JM, Ooguri H, Oz Y. Large N field theories, string theory and gravity. Phys Rep (2000) 323:183-386. doi:10.1016/S03701573(99)00083-6

3. Belhaj A, Chabab M, Moumni HE, Medari L, Sedra MB. The thermodynamical behaviors of kerr-newman AdS black holes. Chin Phys Lett (2013) 30:090402. doi:10.1088/0256-307X/30/9/090402

4. Belhaj A, Chabab M, El Moumni H, Sedra MB. On thermodynamics of AdS black holes in arbitrary dimensions. Chin Phys Lett (2012) 29:100401. doi:10. 1088/0256-307X/29/10/100401

5. Chabab M, El Moumni H, Iraoui S, Masmar K, Zhizeh S. More insight into microscopic properties of RN-AdS black hole surrounded by quintessence via an alternative extended phase space. Int J Geomet Methods Mod Phys (2018) 15(10):1850171. doi:10.1142/S0219887818501712

6. Kubiznak D, Mann RB. P-V criticality of charged AdS black holes. JHEP (2012) 7:033. doi:10.1007/JHEP07(2012)033

7. Kastor D, Ray S, Traschen J. Enthalpy and the mechanics of AdS black holes. Class Quant Grav (2009) 26:195011. doi:10.1088/0264-9381/26/19/195011

8. Dolan BP. Pressure and volume in the first law of black hole thermodynamics. Class Quant Grav (2011) 28:235017. doi:10.1088/0264-9381/28/23/235017

9. Kiselev VV. Quintessence and black holes. Class Quant Grav (2003) 20:1187. doi:10.1088/0264-9381/20/6/310

10. Thomas BB, Saleh M, Kofane TC. Thermodynamics and phase transition of the Reissner-Nordström black hole surrounded by quintessence. Gen Relat Gravit (2012) 44:2181-2189. doi:10.1007/s10714-012-1382-z

11. Li GQ. Effects of dark energy on P-V criticality of charged AdS black holes. Phys Lett B (2014) 735:256-260. doi:10.1016/j.physletb.2014.06.047

12. Wu CH, Zou DC, Wang Y. P-V criticality of born-infeld AdS black holes surrounded by quintessence. Commun Theor Phys (2018) 70(4):459. doi:10. 1088/0253-6102/70/4/459

13. Chabab M, Iraoui S. Thermodynamic criticality of d-dimensional charged AdS black holes surrounded by quintessence with a cloud of strings background. Gen Relat Gravit (2020) 52(8):75. doi:10.1007/s10714-020-02729-4

14. Hilfer R. Applications of fractional calculus in physics. Singapore: World Scientific (2000).

15. Hilfer R. Thermodynamic scaling derived via analytic continuation from the classification of Ehrenfest. Phys Scripta (1991) 44(4):321. doi:10.1088/00318949/44/4/002

16. Hilfer R. Multiscaling and the classification of continuous phase transitions. Phys Rev Lett (1992) 68(2):190-192. doi:10.1103/PhysRevLett.68.190

17. Hilfer R. Scaling theory and the classification of phase transitions. Mod Phys Lett B (1992) 6(13):773. doi:10.1142/S0217984992000855

18. Nagle JF. Lipid bilayer phase transition: density measurements and theory. Proc Natl Acad Sci USA (1973) 70(12):3443-3444. doi:10.1073/pnas.70.12.3443

19. Agnihotri RC, Mehrotra C, Gupta VD, Srivastava V. Fractional order phase transition in lipid bilayer: dipalmitoyllecithin. Pramana - J Phys (1982) 19(1):43-49. doi:10. 1007/BF02846230
20. Riess AG, Filippenko A, Challis P, Clocchiatti A. Observational evidence from supernovae for an accelerating universe and a cosmological constant. Astron J (1998) 116:1009. doi:10.1086/300499

21. Perlmutter S, Aldering G, Goldhaber G, Knop RA, Nugent P, Castro PG, et al. Measurements of Omega and Lambda from 42 high redshift supernovae. Astrophys J (1999) 517:565-586. doi:10.1086/307221

22. Caldwell RR, Dave R, Steinhardt PJ. Cosmological imprint of an energy component with general equation of state. Phys Rev Lett (1998) 80: 1582-1585. doi:10.1103/PhysRevLett.80.1582

23. Podlubny I. Fractional differential equations. San Diego, CA: Academic Press (1999).

24. Caputo M. Linear models of dissipation whose $\mathrm{Q}$ is almost frequency independent-II. Geophys J (1967) 13(5):529-539. doi:10.1111/j.1365-246X. 1967.tb02303.x

25. Chamblin A, Emparan R, Johnson CV, Myers RC. Charged AdS black holes and catastrophic holography. Phys Rev D (1999) 60:064018. doi:10.1103/ PhysRevD.60.064018

26. Gunasekaran S, Mann RB, Kubiznak D. Extended phase space thermodynamics for charged and rotating black holes and Born-Infeld vacuum polarization. JHEP (2012) 11:110. doi:10.1007/JHEP11(2012)110

27. Wheeler JT. Symmetric solutions to the gauss-bonnet extended einstein equations. Nucl Phys B (1986) 268:737-746. doi:10.1016/0550-3213(86)90268-3

28. Wiltshire DL. Spherically symmetric solutions of einstein-maxwell theory with a gauss-bonnet term. Phys Lett B (1986) 169:36-40. doi:10.1016/0370-2693(86) 90681-7

29. Cai RG, Cao LM, Li L, Yang RQ. P-V criticality in the extended phase space of Gauss-Bonnet black holes in AdS space. JHEP (2013) 9:5. doi:10.1007/ JHEP09(2013)005

30. Belhaj A, Chabab M, El moumni H, Masmar K, Sedra MB. Maxwell's equalarea law for Gauss-Bonnet-Anti-de Sitter black holes. Eur Phys J C (2015) 75(2):71. doi:10.1140/epjc/s10052-015-3299-7

31. Kerr RP. Gravitational field of a spinning mass as an example of algebraically special metrics. Phys Rev Lett (1963) 11:237-238. doi:10.1103/PhysRevLett. 11.237

32. Boyer RH, Lindquist RW. Maximal analytic extension of the Kerr metric. J Math Phys (1967) 8:265. doi:10.1063/1.1705193

33. Carter B. Hamilton-Jacobi and Schrodinger separable solutions of Einstein's equations. Commun Math Phys (1968) 10(4):280-310. doi:10.1007/ BF03399503

34. Chabab M, Iraoui S, [arXiv:2010.10931 [hep-th]].

Conflict of Interest: The authors declare that the research was conducted in the absence of any commercial or financial relationships that could be construed as a potential conflict of interest.

Copyright (C) 2021 Chabab and Iraoui. This is an open-access article distributed under the terms of the Creative Commons Attribution License (CC BY). The use, distribution or reproduction in other forums is permitted, provided the original author(s) and the copyright owner(s) are credited and that the original publication in this journal is cited, in accordance with accepted academic practice. No use, distribution or reproduction is permitted which does not comply with these terms. 